Martínez Biot, Bàrbara.

Doctoranda en la Facultad de Bellas Artes de la Universidad Politécnica de Valencia. Departamento de pintura, programa de doctorado en Arte: Producción e investigación.

\title{
Prácticas artísticas colaborativas en la ciudad de Valencia.
}

\section{Collaborative artistic practices in the city of Valencia.}

\author{
TIPO DE TRABAJO: \\ Comunicación. \\ PALABRAS CLAVE: \\ Arte público, ciudad, protesta ciudadana.
}

KEY WORDS:

Public art, city, citizen protest.

RESUMEN.

En la ciudad de Valencia durante las últimas décadas del siglo XX y los primeros del siglo XXI, han florecido una serie de movimientos sociales y plataformas vecinales en respuesta a una serie de problemáticas locales. En un principio, estas plataformas reivindicativas se servían de acciones llamativas para dar a conocer sus reivindicaciones. Dentro de estos casos encontraríamos a Salvem el Botànic o Salvem el Cabanyal que, junto a otros ejemplos, se han servido de acciones artísticas o festivas para visibilizar la crisis social de algunos barrios de la ciudad. Estas acciones favorecían un seguimiento mediático, sin embargo con la irrupción de las nuevas tecnologías el seguimiento mediático se fue debilitando en beneficio de un nuevo protagonismo e independencia. En la actualidad, podemos observar como determinadas plataformas reivindicativas tienen su propio espacio y poder de convocatoria a través de las redes sociales. Esto supone una nueva manera de entender el activismo político, la protesta social y la reivindicación artística; podemos hablar de una nueva forma de comunicación más abierta y eficaz, aunque también desde otro punto de vista podría calificarse también como superficial.

\section{ABSTRACT.}

In the city of Valencia during the last decades of the 20th century and the first of the 21st century, a series of social movements and neighborhood platforms have flourished in response to a series of local problems. Initially, these platforms of protest took advantage of striking actions to make their claims known. In these cases we would find Salvem el Botànic or Salvem el Cabanyal, who, along with other examples, have used artistic or festive actions to make the social crisis of some of the city's districts visible. These actions favored a media monitoring, however with the irruption of the new technologies the media monitoring was weakened in benefit of a new protagonism and independence. At present, we can observe how certain platforms of protest have their own space and power of call through the social networks. This is a new way of understanding political activism, social protest and artistic protest; we can talk about a new form of communication more open and effective, but also from another point of view could also be described as superficial.

\section{CONTENIDO.}

La ciudad de Valencia ha visto florecer en la década final del siglo XX una serie de plataformas reivindicativas como respuesta activa frente a conflictos de carácter territorial y urbanístico. Una creciente desconfianza de la bondad de los proyectos, mayor conciencia ambiental por parte de la población, la pérdida de poder institucional de la izquierda y la llegada de la derecha al gobierno autonómico dieron lugar a movimientos urbanos y rurales de defensa del territorio, el medio urbano y la calidad de vida. Las movilizaciones que 
han llevado a cabo estos colectivos han sido mayoritariamente defensivas, intentando establecer nuevas formas de participación ciudadana y de diálogo.

Aunque los grupos que analizaremos tienen intereses diferentes que van desde la lucha por el territorio hasta las reivindicaciones sociales de colectivos marginados, hay algunas características básicas que podríamos aplicar a todos ellos. Participan personas vinculadas a partidos políticos aunque mayoritariamente sus miembros no lo están, es la independencia política la que les permite atraer a un gran número de adhesiones de sectores muy amplios y libertad de acción. Se aprovechan los conocimientos de especialistas de diferentes campos pero no hay una estructura jerárquica, la participación y la discusión son herramientas fundamentales de trabajo. Estos movimientos deben trabajar a diferentes niveles para garantizar su éxito o al menos un mínimo de efectividad; vemos tres bloques, las acciones de información, las acciones judiciales y las manifestaciones culturales. Estas últimas serán el eje común de estudio de los grupos de protesta que se han dado en la ciudad de Valencia durante los últimos años, estas manifestaciones culturales se producen en el espacio público, generalmente en las calles y espacios problematizados.

Se intenta atraer a un gran número de público no específico para dar visibilidad al problema, estas acciones de carácter artístico y reivindicativo han tenido una larga presencia a lo largo de los años setenta y ochenta en Valencia, y como luego veremos se continúan a finales del siglo XX y principios del XXI. Algunas de estas manifestaciones críticas podemos encontrarlas ya en Valencia, en los años sesenta y setenta con la formación del grupo gráfico Estampa Popular el que producía una serie de calendarios de influencia pop pero sus ilustraciones reflejaban una manera de ver la sociedad franquista crítica y sarcástica.

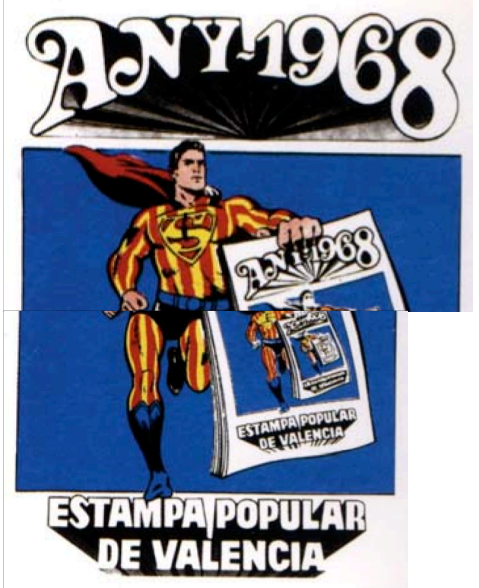

Estampa Popular, portada del calendario de 1968.

Por otra parte, llegados los años noventa las propuestas artísticas evolucionarán hacia formas más alternativas, ejemplos como el grupo de okupación Kasal Popular abren sus críticas al sistema con convocatorias de participación; de otras formas más anónimas y de intervención públicas en Valencia surgen ante problemas concretos y reivindicaciones sociales, como por ejemplo la difusión de servilletas impresos creados por M. Soullard y la Asociación de Vecinos de la Boatella.

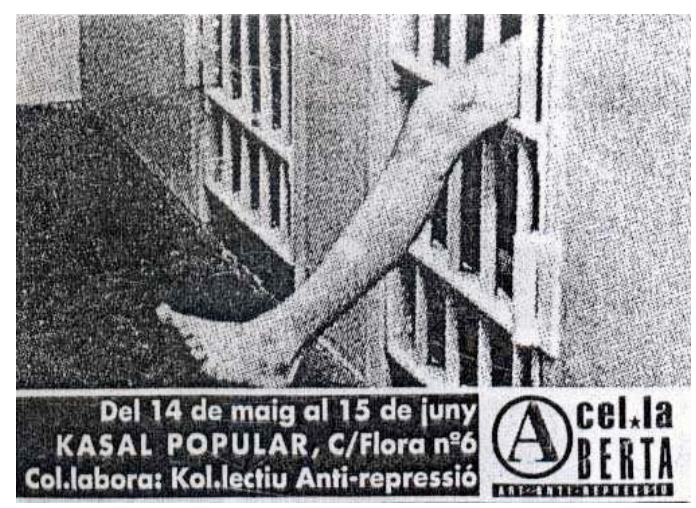

A cel·la oberta, autor desconocido, años del siglo XX. 


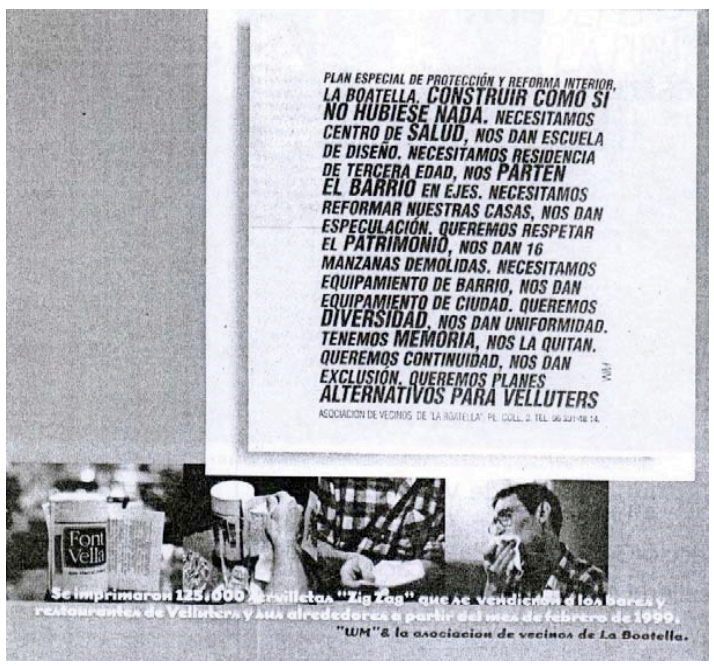

Servilletas Zig-zag, M. Soullard.

La coordinadora ciudadana Salvem el Botànic, Recuperem Ciutat ${ }^{1}$ se presentó el 6 de marzo de 1995, después de que el Ayuntamiento de Valencia aprobara, la construcción de tres edificaciones de 20 alturas en los terrenos del antiguo patio de juegos y de entrada al Colegio de los Jesuitas, junto al Jardín Botánico, en la esquina de la Gran Vía Fernando el Católico y el Paseo de la Pechina. El objetivo de la plataforma es preservar el entorno del Jardín, joya científica reconocida mundialmente, y conservar el perfil urbano del centro histórico de Valencia, patrimonio paisajístico de la ciudad y de todo el pueblo valenciano. Constituida sin ánimo de lucro ni adscripción política por un grupo variable de personas de diferentes profesiones, una de las características más reseñables de su funcionamiento ha sido el apoyo generalizado que ha recibido de todo tipo de instituciones, colectivos y estamentos sociales y culturales. Algunas de las acciones reivindicativas más conicidas y por ende eficaces son:

Campaña de fin de año, padres Navidades a las puertas de las empresas de Lladró y de Miguel, en Expo-Hotel, en el Ayuntamiento de Valencia y al borde de los terrenos amenazados. Diciembre de 1995 hasta 2004.

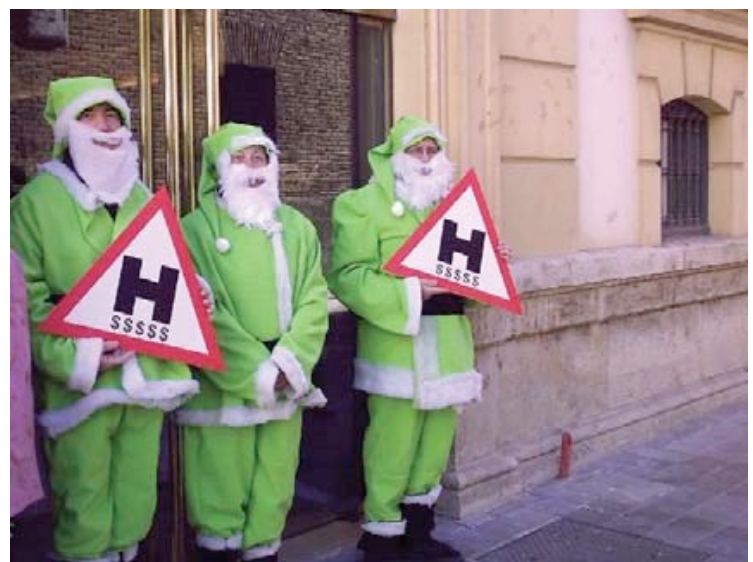

Papàs Noel a la puerta de una de las empresas implicadas, 1995.

Los padres Noel verdes de la coordinadora Salvem el Botànic se presentaron en repetidas ocasiones a las puertas del Ayuntamiento de Valencia para recordar a la alcaldesa Rita Barberá la amenaza que supondría para el jardín la construcción del hotel previsto en el solar de Jesuitas. Con una señal de tráfico que indicaba precaución, los miembros de la coordinadora presentaron un memorando a la sociedad valenciana donde detallan "las escandalosas cifras de crecimiento injustificado del proyecto" ${ }^{2}$

\footnotetext{
${ }^{1}$ MILLET, Teresa (Ed.), Estampa Popular, Valencia, Generalitat Valenciana, 1996.

2 "Los Papá Noel de Salvem el Botànic visitaron a la alcaldesa Barberá", El País C. Valenciana, 30-12-2004.
} 


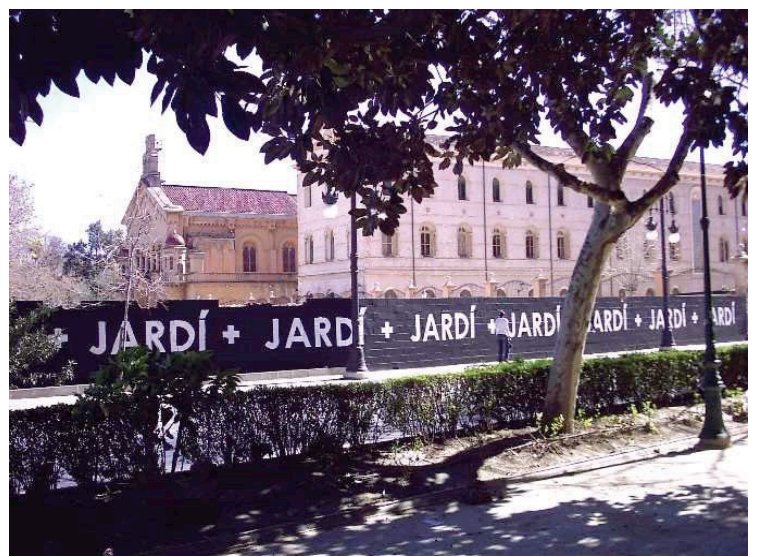

Jardí + Jardí +... año 2000.

Profesores y alumnos de la Facultad de Bellas Artes de Valencia utilizaron grandes murales para combatir la intención constructora del Ayuntamiento y exigir que el jardín se extienda hacia el solar que se encuentra junto al colegio de los Jesuitas. Estudiantes de Biológicas y parejas jóvenes con hijos, algunos políticos y artistas se dieron cita entre acordes de tabalet y dolçaina y en un ambiente lúdico con dibujos de inspiración infantil en un mural de estilo abstracto a base de grandes manchas verdes y azuladas, todo sirvió para reclamar la protección del jardín ${ }^{3}$.

Cadena humana (abrazo) alrededor de Jesuitas. 15 de diciembre de 2002.

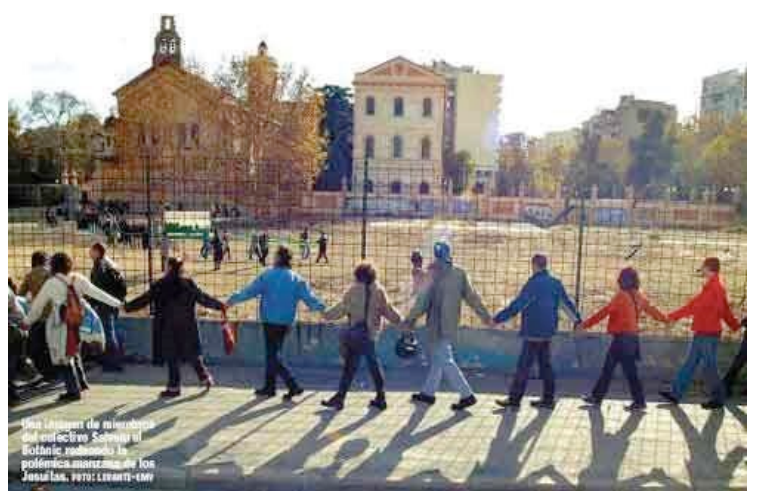

Abrazo reivindicativo, año 2002.

La plataforma cívica retomó las movilizaciones para conseguir que una de las fachadas históricas de la ciudad que da al Oeste sobre el viejo cauce del río Turia recuperara su esplendor. Cientos de ciudadanos rodearon simbólicamente la isla donde se sitúa el solar de Jesuitas en un abrazo como una medida reivindicativa que tuvo éxito en países como Italia y supuso una vuelta simbólica a los inicios de unas movilizaciones que duraban ya nueve años. Un espacio donde ahora se puede visitar uno de los jardines más bonitos de Valencia, el de las Hespérides, separado sólo por una estrecha calle del jardín Botánico de la Universidad de València ${ }^{4}$.

En paralelo a las protestas por el Botànic, la coordinadora cívica Defensem La Punta Salvem I'Horta, constituida el día 13 de enero de 1998, llevó a cabo una exposición de fotografías sobre la Huerta de la Punta. Esta campaña incluyó, además de la exposición mencionada, una serie de mesas redondas con proyección de diapositivas explicativas de carácter pedagógico, así como diferentes acciones testimoniales y manifestaciones. Expusieron un total de 48 fotografías, 3 por autor/a. La exposición tuvo un carácter itinerante y se mostró en aquellos barrios o poblaciones que de una manera u otra se han visto afectados por la especulación urbanística y la desaparición de la huerta. También se realizaron bonos de ayuda a la coordinadora, numerados, con un importe de

\footnotetext{
${ }^{3}$ PRATS, Jaime, "Arte para salvar el Botànic", El País C.Valenciana [en línea], [consulta 22 de febrero de 2017] disponible en $<$ elpais.com>.

${ }^{4}$ FERRANDIS, J., “Un abrazo y volvemos a empezar", El País C.Valenciana [en línea], [consulta 25 de febrero de 2017] disponible en $<$ elpais.com>.
} 
300 pesetas que daban opción a participar en el sorteo de las fotografías el día de la clausura de la exposición. Prestaron su colaboración: Asociación de vecinos La Unificadora de la Punta, Centro Excursionista de Valencia, Departamento de Escultura UPV, Fundación Bancaja-Sagunto. Expusieron sus fotografías las siguientes personas: Nadia Collette, Pablo Lorenzo, Mariví Jiménez, Jose Māa Azcárraga, Cesar Soler, Luisa Alvaro, Tato y Caso, Mateo Gamón, Rafael Garay, Juan Peiró, Carmen Soler, Pedro Magana, Emilio Roca, Quique Pascual, Adolf Herrero, Karina Sánchez. ${ }^{5}$

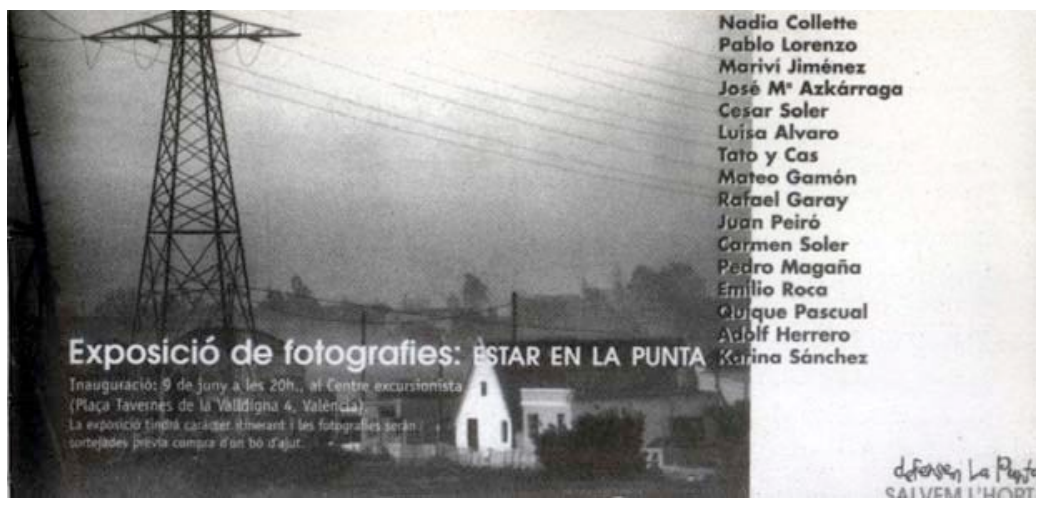

Invitación a la inauguración oficial de Estar en la Punta, año1998

PATIRMONI PATIRMONI. Mayo de 2003.

Fue una acción artística efímera, coordinada por el creador catalán Frederic Perers, que combinaba las palabras y la tierra, la fuerza de su significado y la energía de la Huerta de la Punta que las contenía. Una especie de poesía visual a gran escala escrita sobre latTierra. La plataforma Per l'Horta organizó esta acción artística como homenaje a esta partida de la Huerta de Valencia y su gente, cuando la destrucción de La Punta era inminente. Se hizo una serie de 100 fotografías aéreas numeradas y firmadas por Frederic Perers, que documentan la acción. SUFRIR + PATRIMONIO = PATIRMONI ${ }^{6}$.

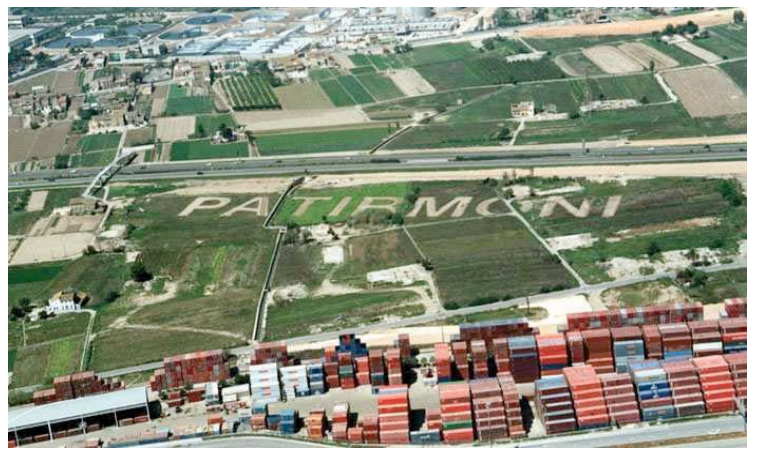

Imagen aérea de la acción Patirmoni, año 2003.

Una vez más asistimos a la eterna confrontación: entre la conservación del patrimonio o el "desarrollo especulativo" de una ciudad. Desde el siglo pasado los habitantes del Cabanyal han vivido con la amenaza de la prolongación de la Avenida Blasco Ibáñez, hasta que con la construcción de la estación de RENFE en la confluencia con la Avenida de Serrería, se creía que aquí se finalizaba la historia de una Avenida-Paseo, que tenía su inicio-final en los jardines de Viveros y su final-inicio en la estación de RENFE y en el barrio del Cabanyal. Pero en el Pleno del Ayuntamiento de Valencia del día 24 de Julio de 1998, el Partido Popular, que gozaba de mayoría y en contra de las otras fuerzas políticas, aprobó el anteproyecto de Prolongación de la Avenida Blasco lbáñez hasta el mar.

Entre 1998 y 2005 Cabanyal Portes Obertes $^{7}$, fue un proyecto de intervenciones artísticas, surgió ante la grave amenaza para la supervivencia del barrio del Cabanyal de Valencia que suponían los planes urbanísticos del Ayuntamiento. Estaba organizado por un amplio colectivo de artistas agrupados en la plataforma Salvem el Cabanyal-Canyamelar, comprometidos e implicados con la lucha

\footnotetext{
${ }^{5}$ ALBELDA, J.L., Estar en La Punta: retrato de una exposición itinerante, Valencia, Editorial UPV, 2002.

${ }^{6}$ FREDERIC PERERS, <http://www.fredericperers.cat/index1.html>, [consulta 6 de marzo de 2017].

${ }^{7}$ MARTÍNEZ, Emilio (Ed.), Cabanyal Portes Obertes. Art, política i participació ciudadana, Valencia, Plataforma Salvem El CabanyalCanyamelar, 2003.
} 
social que se desarrollaba en este barrio. Este era un proyecto de convocatoria abierta, a todos aquellos artistas que quisieran manifestarse ante el abuso de poder y las prácticas antidemocráticas que conlleva la especulación inmobiliaria que se estaba desarrollando en nuestras ciudades y en esos momentos en el barrio del Cabanyal, como resultado de las prácticas neoliberales en las sociedades actuales. En cada una de las ediciones de Cabanyal Portes Obertes se han realizado entre 160 y 200 proyectos de artes plásticas, música, fotografía, vídeo, performance, teatro y danza. En las calles del barrio, en los tres teatros que existen actualmente en el Cabanyal, y especialmente en las casas particulares de los vecinos que abrían sus puertas al público que asistía a estas jornadas.

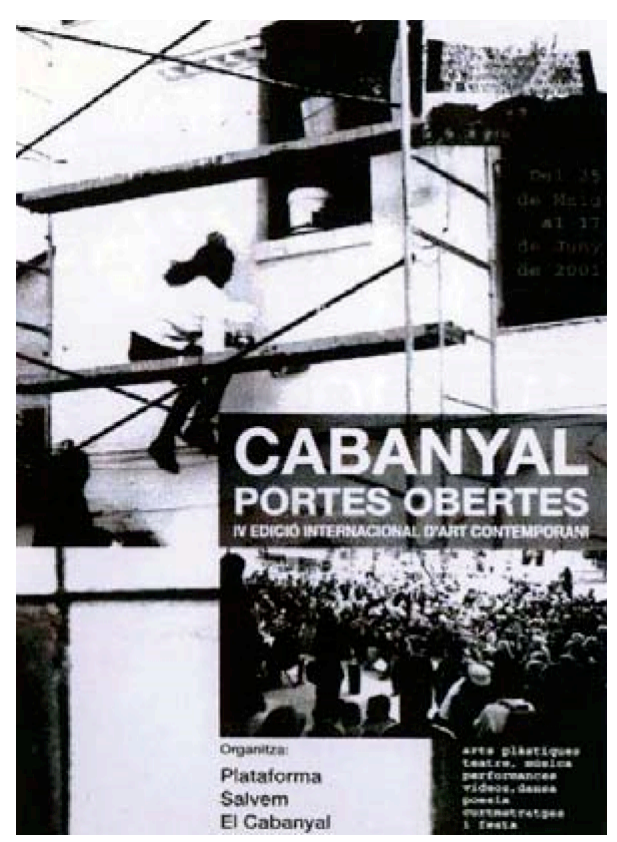

Cartel anunciador de Cabanyal Portes Obertes, año 2001.

Este era un proyecto autogestionado, voluntario, espontáneo, financiado por los propios vecinos, los pequeños comerciantes del barrio, los artistas, cada uno aporta lo dispuesto, sus casas, su tiempo, su trabajo. Inicialmente el objetivo de estas jornadas era que los ciudadanos de Valencia conocieran la realidad del barrio y la amenaza de desaparición que se ciernen sobre él. De los primeros 5000 espectadores de la edición del 98 hasta la última edición el incremento de público es uno de los hechos decisivos que animan a los organizadores y los vecinos a continuar organizando estas jornadas. Una de las características principales es que las intervenciones realizadas por los artistas se exponían en las calles del barrio y en las casas de los vecinos, que abrían sus puertas al público todos los fines de semana durante el mes que duraba esta manifestación. Esta relación entre lo público y lo privado, las intervenciones artísticas y el contexto cotidiano de cada casa, los artistas, los vecinos y los espectadores, aproximan arte y vida en un momento común como muy pocas veces hemos tenido oportunidad de contemplar.

La asociación de vecinos y comerciantes Amics del Carme se constituyó el mes de mayo de 2000 con el objetivo de responder a una serie de problemas que son comunes a otros barrios del centro histórico de Valencia y de otras ciudades europeas, tales como la especulación urbanística, la despoblación, la degradación de edificios históricos, la pérdida del tejido social y comercial, y la falta de las infraestructuras necesarias para responder a las necesidades básicas de los vecinos. En pocas palabras, el Carmen se está transformando en un parque temático de copas durante la noche ${ }^{8}$. ¿Pintamos algo? es una acción directa que nace de la necesidad de expresarse y mejorar el entorno degradado dando curso a los anhelos creativos: murales, graffitis, acciones, instalaciones, percusión y músicas, software libre, poesía, malabares... en los entornos de la calle Alta del Carmen. Muro de la Resistencia estaba pensada como una más dentro del plan ¿Pintamos algo? El proyecto consistía en colocar una gran tela con grandes fotografías de referencia a la situación local y global, más artículos de prensa, más los escritos recibidos desde diferentes lugares del mundo. De esta manera cualquier persona podía escribir lo que le diera la gana: desde un poema a un grito o denuncia o expresión de amor o lucha o patada.

\footnotetext{
${ }^{8}$ ASSOCIACIÓ DE VEİNS AMICS DEL CARME, < www.amicsdelcarme.com/>, [consulta 11 de marzo de 2017].
} 


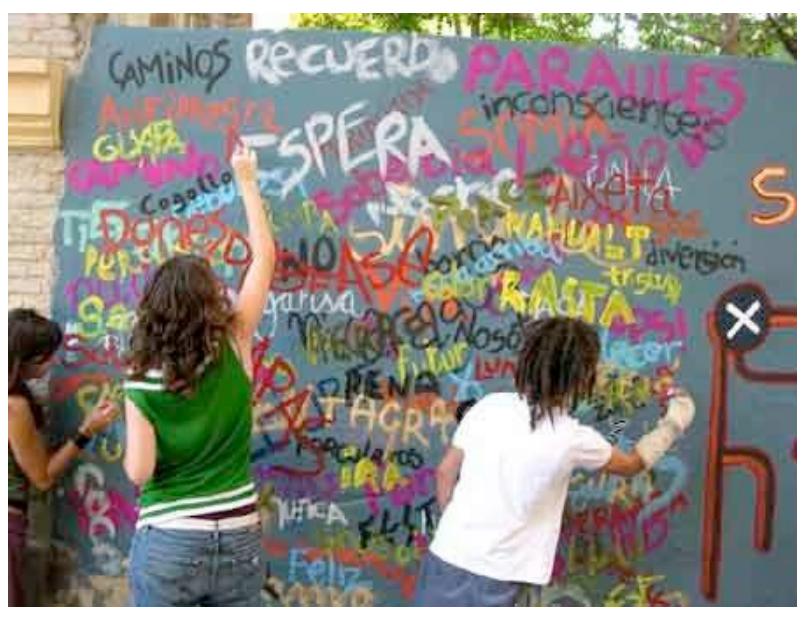

Trabajo colectivo en el mural del barrio de El Carme, 2003.

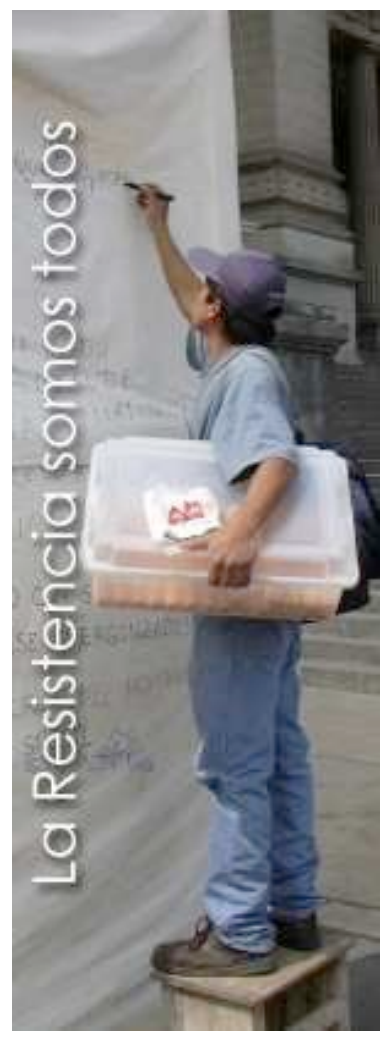

Proceso de El muro de la resistencia, 2003.

En conclusión, esta pequeña muestra de las prácticas artísticas colaborativas en la ciudad de Valencia nos descubre un tejido asociativo de carácter reivindicativo que protagonizó un momento determinado de la historia reciente de la ciudad, en algunos casos obteniendo resultados positivos como ha sido el caso del Botànic y el Cabanyal; por otro, negativos como la problemática de la ZAL en la Punta y otros ejemplos que han quedado recogidos en otras investigaciones recientes. La irrupción de estas plataformas no puede dejar de entenderse tampoco junto con las acciones artísticas que las acompañado e incluso visibilizado de forma eficaz, cabe destacar la participación de las instituciones universitarias de la ciudad como espacios de exposición, difusión y debate que han dado lugar a un amplio número de reflexiones teóricas y trabajos artísticos de gran valor patrimonial para Valencia y su población. 